\title{
Complimentary Microscopic Techniques and Computational Fluid Dynamic Analysis of Blood Flow in a Medical Device
}

\author{
M.A. Murphy, J.P. DiOrio, L. Stojanovic, A. Yardimci, J. DelCastillo, Chen, S.
}

Technology Resources, Baxter Healthcare, State Route 120 \& Wilson Road, Round Lake, Illinois 60073

Biocompatibility assessment of medical devices or medial device components that are in direct blood contact is an essential element in product development for the medical device industry. Correlative microscopic methods along with computational fluid dynamics (CFD) modeling are valuable tools for this type of assessment. This paper describes these methods and others used to analyze experimental filtration membranes and blood set connections following whole blood exposure.

The filtration membrane was exposed to heparinized human blood. The blood set connectors were placed in an "in vitro loop" perfusion system containing the test articles and lightly anti-coagulated (sodium citrate) whole human blood. Specifically, pressure changes across the areas of interest were monitored. Samples for scanning electron microscopy (SEM) were fixed in 2\% glutaraldehyde, dehydrated through a graded $0-100 \%$ ethanol series and air-dried out of hexamethyldisilazane. They were coated with palladium and imaged on a JEOL 6300 field emission SEM operated at $5 \mathrm{kV}$. Samples for transmission electron microscopy (TEM) were fixed in 2\% glutaraldehyde osmicated followed by dehydration through a graded $0-100 \%$ ethanol series into acetone and embedment in Spurr's resin. Thin sections were stained with uranyl acetate and lead citrate and were imaged on a JEOL 1200 EX TEM at operated at $60 \mathrm{kV}$. Blood connector parts were also examined prior to blood exposure using a Veeco Wyko Profiling System NT3300. These parts were then coated with palladium and imaged on a JEOL 6300 field emission SEM operated at 5kV.

An axis-symmetric model of the flow domain was generated from drawings and dimensional data for the connector system. Commercially available mesh generation software was used to create the computational mesh. Working fluid (blood) was used in the simulation with relevant physical parameters for $40 \mathrm{Hct}$ blood at $30^{\circ} \mathrm{C}$. An inlet boundary condition was employed resulting in a processing rate of $85 \mathrm{ml} / \mathrm{min}$. Appropriate outlet, symmetry, and wall boundary conditions were employed in the simulation. The flow was simulated in a laminar, steady-state, Newtonian configuration observing relevant non-dimensional parameters.

In the SEM (Figure 1), a layer of spread platelets was seen on the filtration membrane with an associated layer of dendritic/pseudopodial platelets. In the TEM (Figure 2), the membrane is seen in cross-section which shows the thickness of the spread platelet layer with an associated platelet. This is consistent with the platelet literature which shows that a base layer of fully spread platelets can underlie platelet surface aggregates [1]. In prescreening of the blood connector parts, a ridge of approximately $65 \mu \mathrm{m}$ in height on the top of the blood connector was seen as shown by profilometry (Figure 3) and SEM. When exposed to blood, clot material was found and is shown associated with this ridge (Figure 4). The CFD model of the blood connector predicted a stagnation and recirculation zone next to the wall of the part (Figure 5). When this part was tested in the "in vitro loop" system, a clot was seen in the same area (Figure 6). 
References

[1] S. Goodman, J. Biomed. Mat Res. 45 (1999) 249-250.
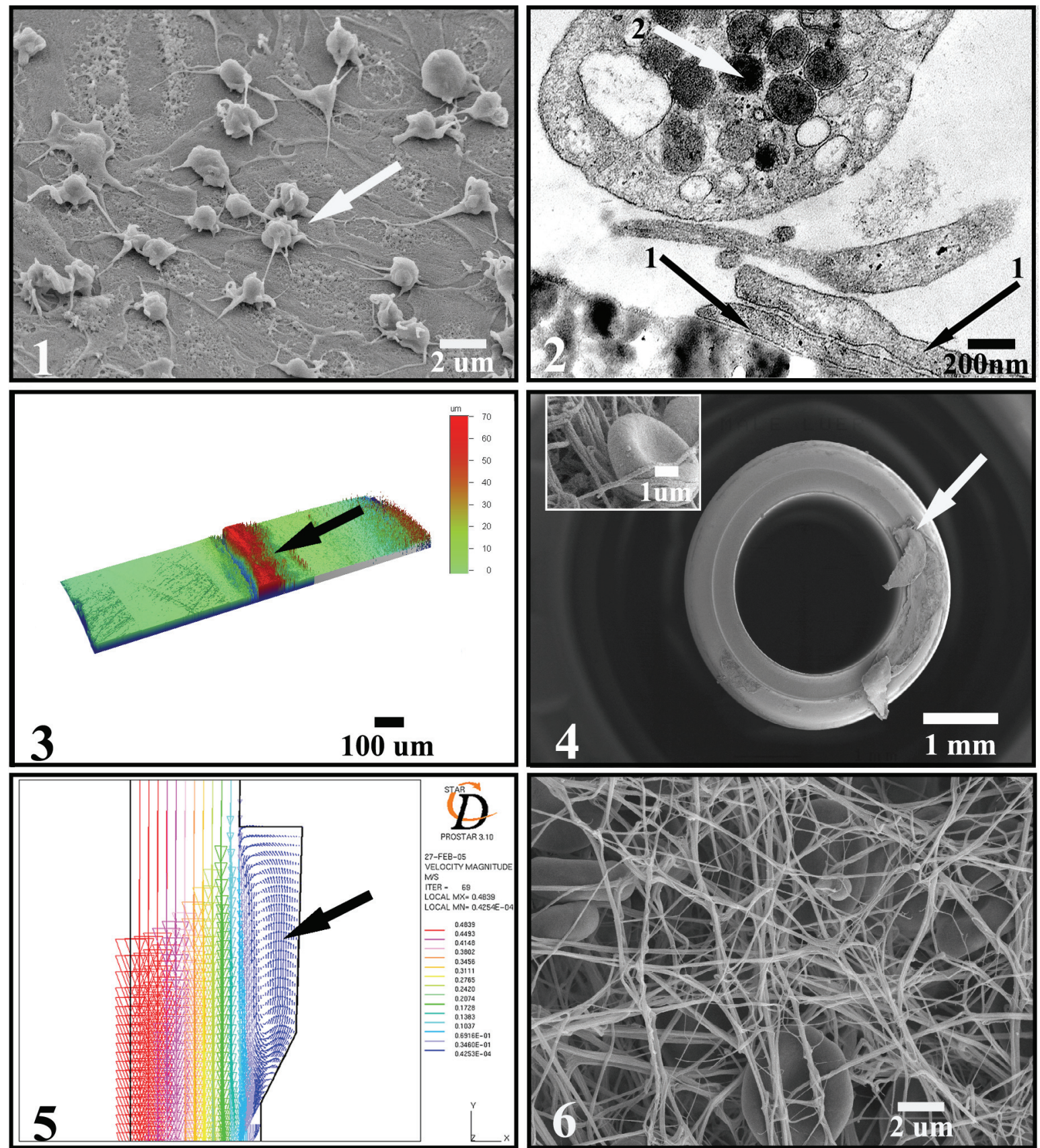

Fig. 1. SEM of platelet layers on a membrane surface. Arrow at granule in associated platelet.

Fig. 2. TEM of a cross-section in a similar area as Figure 1. Arrow 1 at spread platelet on the filtration membrane surface. Arrow 2 at centralized granule.

Fig. 3. Profilometry of a prescreened connector showing ridge at arrow.

Fig. 4. SEM of a similar part as Figure 3 after blood contact. Clot Material at ridge (arrow) in inset.

Fig. 5. CFD of the blood connector, arrow at the stagnation and recirculation zone.

Fig. 6. SEM of a similar area in Figure 5 following blood contact and showing clot material. 